\title{
Non-equivalent role of TM2 gating hinges in heteromeric Kir4.1/Kir5.1 potassium channels
}

\author{
Lijun Shang $\cdot$ Stephen J. Tucker
}

Received: 11 April 2007 / Revised: 20 June 2007 / Accepted: 27 June 2007 / Published online: 27 July 2007

(C) EBSA 2007

\begin{abstract}
Comparison of the crystal structures of the KcsA and MthK potassium channels suggests that the process of opening a $\mathrm{K}^{+}$channel involves pivoted bending of the inner pore-lining helices at a highly conserved glycine residue. This bending motion is proposed to splay the transmembrane domains outwards to widen the gate at the "helix-bundle crossing". However, in the inwardly rectifying (Kir) potassium channel family, the role of this "hinge" residue in the second transmembrane domain (TM2) and that of another putative glycine gating hinge at the base of TM2 remain controversial. We investigated the role of these two positions in heteromeric Kir4.1/Kir5.1 channels, which are unique amongst Kir channels in that both subunits lack a conserved glycine at the upper hinge position. Contrary to the effect seen in other channels, increasing the potential flexibility of TM2 by glycine substitutions at the upper hinge position decreases channel opening. Furthermore, the contribution of the Kir4.1 subunit to this process is dominant compared to Kir5.1, demonstrating a nonequivalent contribution of these two subunits to the gating process. A homology model of heteromeric Kir4.1/Kir5.1 shows that these upper "hinge" residues are in close contact with the base of the pore $\alpha$-helix that supports the selectivity filter. Our results also indicate that the highly conserved glycine at the "lower" gating hinge position is required for tight packing of the TM2 helices at the helix-bundle crossing, rather than acting as a hinge residue.
\end{abstract}

L. Shang $\cdot$ S. J. Tucker $(\bowtie)$

Oxford Centre for Gene Function,

Department of Physiology Anatomy and Genetics,

University of Oxford, Oxford, UK

e-mail: stephen.tucker@physiol.ox.ac.uk

\section{Introduction}

Despite the wealth of high-resolution structural data now available for many different potassium channels, the structural motions that occur as these dynamic proteins switch between the open and closed states is still not clear and presents a considerable challenge in ion channel structurefunction analysis.

Our current understanding of this process is based primarily upon comparison of the X-ray crystal structures of the $\mathrm{KcsA} \mathrm{K}^{+}$channel in the closed state with that of MthK in the open state (Doyle et al. 1998; Jiang et al. 2002). This has led to a structural model of $\mathrm{K}^{+}$channel gating in which the physical gate is formed by the four inner pore-lining TM2 helices, which cross over each other constricting the pore near its intracellular entrance. During channel opening, the TM2 helices are proposed to bend in the middle and splay outwards so that the gate at the lower "helix bundle crossing" widens forming an open pathway from the cytoplasm to the selectivity filter (Fig. 1a; Doyle 2004; Perozo et al. 1999).

In the open MthK structure, the inner helices are bent at a highly conserved glycine residue in TM2 (Gly-83; Jiang et al. 2002). A glycine is found at this position in TM2 in $>80 \%$ of almost 500 different potassium and CNG channel sequences (Magidovich and Yifrach 2004) suggesting that this may be a common mechanism of gating in this architecturally conserved superfamily of ion channels. In support of this, functional studies of several different classes of $\mathrm{K}^{+}$channel, and structurally related $\mathrm{Na}^{+}$channels, have demonstrated that replacing this glycine with residues that reduce TM2 flexibility impair channel gating (Ding et al. 2005; Jin et al. 2002; Magidovich and Yifrach 2004; Zhao et al. 2004). Likewise, in the KCNQ1 channel, which does not have a glycine at this position, substitution of a glycine 
A
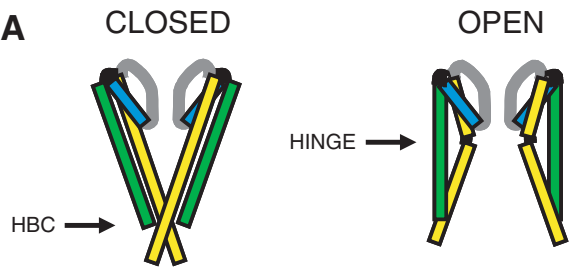

B UPPER LOWER

Kir1.1 TAIFLLIFQSILGVIINSFMCGAILA

Kir2.1 IAVFMVVFQSIVGCIIDAFIIGAVMA

Kir3.1 EGIILFLFQSILGSIVDAFLIGCMFI

Kir4.1 LAIVLLIAQLVLTTILEIFITGTFLA

Kir5.1 VAVLTVILQSILSCIINTFIIGAALA

Kir6.2 LAILILIVQNIVGLMINAIMLGCIFM

Kir7.1 SAIALLAIQMLLGLMLEAFITGAFVA

KcsA WGRCVAVVVMVAGITSFGLVTAALAT

MthK LGMYFTVTLIVLGIGTFAVAVERLLE

shaker WGKIVGSLCAIAGVLTIALPVPVIVS

Fig. 1 a Cartoons of the closed state KcsA (left) and open state MthK (right) structures. The relative positions of the helix-bundle crossing $(H B C)$ and upper glycine hinge (Hinge) in TM2 (yellow) are indicated. b An alignment of TM2 from a range of different mammalian Kir channels with the KcsA, MthK and Shaker sequences. Note that the upper glycine hinge is not conserved in Kir4.1 or Kir5.1, but that the lower glycine "hinge" residue is highly conserved

into this position (A366G) increases the ability of the channel to open (Seebohm et al. 2006). All these results support a model in which bending of TM2 occurs at this "glycine hinge" position and predict that changes which reduce TM2 flexibility impair channel opening, whilst mutations that enhance its flexibility at this hinge position increase channel opening.

However, it has also been suggested that bending of TM2 may occur lower down at the helix-bundle crossing. This "lower" position correlates with the "PVP" motif in the voltage-gated Shaker channel, which is thought to induce a kink in the transmembrane helix (Bright et al. 2002), and the recently published Kv1.2 crystal structure also exhibits an open channel conformation without any apparent bending of S6(TM2) at the upper glycine hinge (Long et al. 2005). Furthermore, in the inwardly rectifying (Kir) channel family, another highly conserved glycine residue is found at this position (Magidovich and Yifrach 2004), suggesting that flexibility of TM2 may also be important at this site.

The relative importance of these "upper" and "lower" glycine hinges to Kir channel gating remains controversial, as several members of the Kir channel family (e.g. Kir4.1 and Kir5.1) do not possess a glycine at the upper hinge position (Fig. 1b), whereas the lower glycine residue is absolutely conserved in all known members of the Kir channel superfamily. The non-conserved nature of the upper hinge in Kir4.1 and Kir5.1 may be structurally and functionally related to the fact that Kir5.1 exhibits highly selective heteromultimerisation with Kir4.1 (and Kir4.2, which also has a non-conserved upper hinge residue; Konstas et al. 2003; Pessia et al. 2001). Heteromeric Kir4.1/ Kir5.1 channels are expressed primarily in renal tubular epithelia and also in certain neuronal populations where they are thought to be involved in $\mathrm{K}^{+}$buffering and recycling (Derst et al. 2001; Wu et al. 2004).

In this study, we have investigated the role of these putative gating hinges in recombinantly expressed heteromeric Kir4.1/Kir5.1 channels. Unlike the effects seen in other channels, we found that increasing the potential flexibility of TM2 by glycine substitutions at the upper hinge position decreased channel opening. Furthermore, the contribution of the Kir4.1 subunit to this process was dominant compared to Kir5.1. We also found that the highly conserved glycine at the "lower" gating hinge position is probably required for tight packing of the TM2 helices at the helixbundle crossing, rather than acting as a hinge residue.

\section{Methods}

In order to accurately compare any differences in intrinsic channel properties and expression levels caused by these mutations, we chose the Xenopus oocyte expression system. All channel subunits were subcloned into the oocyte expression vector $\mathrm{pBF}$, which provides $5^{\prime}$ and $3^{\prime}$ untranslated regions from the Xenopus $\beta$-globin gene flanking a polylinker containing multiple restriction sites. In all cases where the heteromeric channels were studied, the Kir subunits were expressed as Kir4.1-Kir5.1 dimers linked at tandem. This well-established method produces heteromeric channels with the correct stoichiometry (2:2) and arrangement (alternate Kir4.1 and Kir5.1 subunits) and does not alter the intrinsic biophysical properties of the channel (Cui et al. 2001; Pessia et al. 2001, 1996; Yang et al. 2000). Site directed mutagenesis was performed using the QUICKCHANGE XL protocol (Stratagene).

Messenger RNA, encoding both wild-type and mutant heteromeric Kir4.1-Kir5.1 (or homomeric Kir4.1), were in vitro transcribed using the SP6 mMessageMachine system (Ambion). mRNA concentrations were quantified and standardised by spectrophotometric analysis and mRNA quality assessed by electrophoresis and ethidium bromide staining. Equal quantities of either wild-type or mutant mRNAs were then microinjected into Xenopus oocytes ( $0.25 \mathrm{ng} /$ oocyte) according to standard protocols.

Macroscopic whole-cell currents were recorded as done previously (Casamassima et al. 2003; Pessia et al. 2001; Tucker et al. 2000). For cell attached single-channel currents, the pipette solution contained (in $\mathrm{mM}$ ): $\mathrm{KCl} 120, \mathrm{CaCl}_{2}$ 1.8 and HEPES 10 (pH 7.2). The bath solution contained 
(in $\mathrm{mM}$ ): $\mathrm{KCl}$ 120, EGTA 2, Tetrasodiumpyrophosphate 1 and HEPES 10 (pH 7.2). Single-channel activity was recorded using an Axopatch 200B amplifier (Axon Instruments) over a range of different voltages between +40 and $-120 \mathrm{mV}$, filtered at $1-2 \mathrm{kHz}$ (Frequency Devices 900), sampled at $5-10 \mathrm{kHz}$ and stored directly into the computer's hard disk through the Digidata 1322A interface (Axon Instruments). The voltages stated refer to the command potential from the amplifier. All measurements were made at room temperature $\left(21-23^{\circ} \mathrm{C}\right)$. Analysis was carried out with Clampfit 9.2 (Axon Instruments). All statistical values quoted are the mean \pm the standard error. Where indicated, the Student's $t$ test was used to analyse the significant difference between wild-type and mutant channels. In the figures, $P$ values of $*<0.005$ and $* *<0.05$ were considered to be statistically significant.

\section{Results and discussion}

The upper glycine hinge

Wild-type Kir4.1 has a threonine at the upper hinge position, whilst Kir5.1 has a serine. According to several recent studies, introduction of a smaller glycine side chain at these positions is predicted to increase TM2 flexibility and increase the ability of the channel to open (RosenhouseDantsker and Logothetis 2006; Seebohm et al. 2006). We therefore mutated these positions to glycine residues in both Kir4.1 (T154G) and Kir5.1 (S157G) and measured their effect on channel activity in the heteromeric Kir4.1/ Kir5.1 channel.

Contrary to expectation, we found that mutation of the upper TM2 gating hinge residue in both Kir4.1 and Kir5.1 to a glycine residue did not increase channel activity. Instead, Kir4.1(T154G)/Kir5.1 channels exhibited reduced whole-cell currents, which correlated with a reduced singlechannel open probability $\left(P_{\mathrm{o}}\right.$; Fig. 2$)$. Kir4.1/Kir5.1 (S157G) channels were similar to wild-type, whilst the double mutant Kir4.1(T154G)/Kir5.1(S157G) channels were not different from the single Kir4.1(T154G)/Kir5.1 mutation (Fig. 2 and Table 1). For all of these mutants, there was a good correlation between the observed wholecell currents and single-channel open probability (Fig. 2). Furthermore, no reduction in single-channel current amplitude was observed for any of these mutants, or any changes in bursting behaviour (Fig. 3). Instead, single-channel kinetic analysis revealed that the reduction in $P_{\mathrm{o}}$ was principally due to a prolonged mean closed time and a reduced mean open time (Table 1A).

We also tested the effect of the Kir4.1(T154G) mutation in homomeric Kir4.1 channels. The effects observed were similar to those seen in heteromeric Kir4.1(T154G)/Kir5.1 channels with a $57 \pm 3 \%(n=30)$ decrease in whole-cell currents compared to wild-type Kir4.1. This correlated with a reduced $P_{\mathrm{o}}$ of $0.36 \pm 0.04(n=6)$ for $\mathrm{Kir} 4.1(\mathrm{~T} 154 \mathrm{G})$

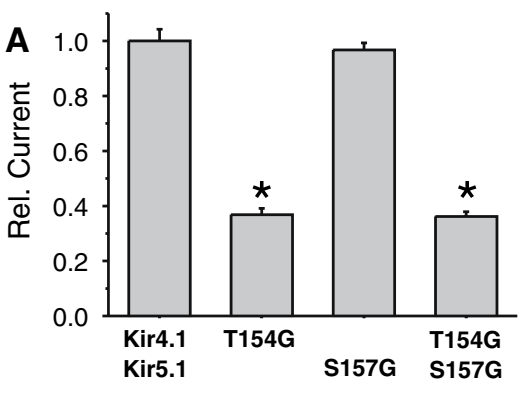

Fig. 2 a Relative whole-cell currents for glycine mutations at the upper hinge position in different subunits of heteromeric Kir4.1/Kir5.1 channels. Maximum steady-state currents were recorded at $-120 \mathrm{mV}$,

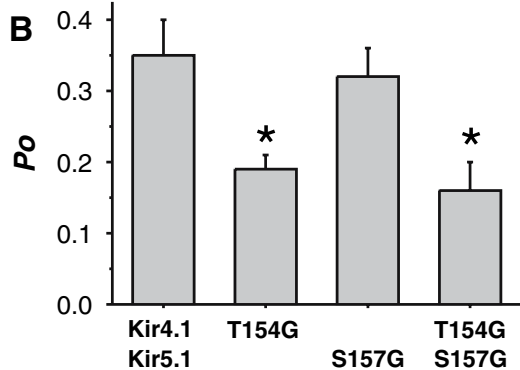

$n=12$. b Single-channel open probability values $\left(P_{\mathrm{o}}\right)$ for the same mutations (see "Methods" and also Table 1). $* P<0.005$
Table 1 Kinetic parameters of mutations at the upper hinge position in A: heteromeric Kir4.1/ Kir5.1, and B: homomeric Kir4.1

MOT mean open time, $M C T$ mean closed time, and $M B D$ mean burst duration. Values are in $\mathrm{ms} ; n=5-8$

\begin{tabular}{lllll}
\hline & Po & MOT & MCT & MBD \\
\hline A & & & & \\
Kir4.1/Kir5.1 & $0.35 \pm 0.05$ & $2.30 \pm 0.01$ & $2.98 \pm 0.01$ & $14.77 \pm 11.0$ \\
Kir4.1(T154G)/Kir5.1 & $0.19 \pm 0.02$ & $1.08 \pm 0.01$ & $4.44 \pm 0.02$ & $8.6 \pm 5.2$ \\
Kir4.1/Kir5.1(S157G) & $0.32 \pm 0.04$ & $1.97 \pm 0.01$ & $2.30 \pm 0.02$ & $14.77 \pm 8.7$ \\
B & & & \\
Kir4.1 & $0.45 \pm 0.05$ & $97.19 \pm 8.95$ & $22.87 \pm 2.39$ & \\
Kir4.1(T154G) & $0.36 \pm 0.04$ & $14.82 \pm 2.75$ & $21.29 \pm 3.68$ & \\
\hline
\end{tabular}




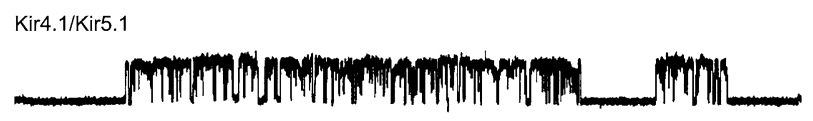

Kir4.1(T154G)/Kir5.1
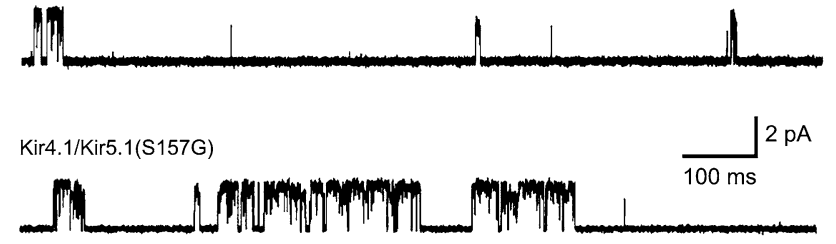

Kir4.1(T154G)/Kir5.1(S157G)

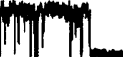

Fig. 3 Representative single-channel currents for wild-type Kir4.1/ Kir5.1, Kir4.1(T154G)/Kir5.1, Kir4.1/Kir5.1(S157G) and Kir4.1 (T154G)/Kir5.1(T157G) mutants recorded in the cell-attached mode at $-120 \mathrm{mV}$. No differences are seen in either the amplitude of the current or the "bursting" single-channel behaviour with multiple subconductance states. However, the open probabilities $\left(P_{0}\right)$ were decreased and correlate with the reduction in whole-cell current (see also Table 1)

compared to $0.45 \pm 0.05(n=6)$ for wild-type Kir4.1. Single-channel kinetic analysis showed that this reduction in $P_{\mathrm{o}}$ was mainly due to a reduced open time (Table 1B).

We next tested the effect of different amino acid substitutions at these upper hinge positions in Kir4.1/Kir5.1. For the Kir4.1 subunit, mutation of Thr-154 to an alanine or a serine had little or no effect on single-channel open probability (Fig. 4), whereas mutation to a valine caused a dramatic reduction in $P_{\mathrm{o}}$. By contrast, for the Kir5.1 subunit, substitution of Ser-157 to a threonine, alanine or valine had no obvious effect on channel $P_{\mathrm{o}}$ (Fig. 4).

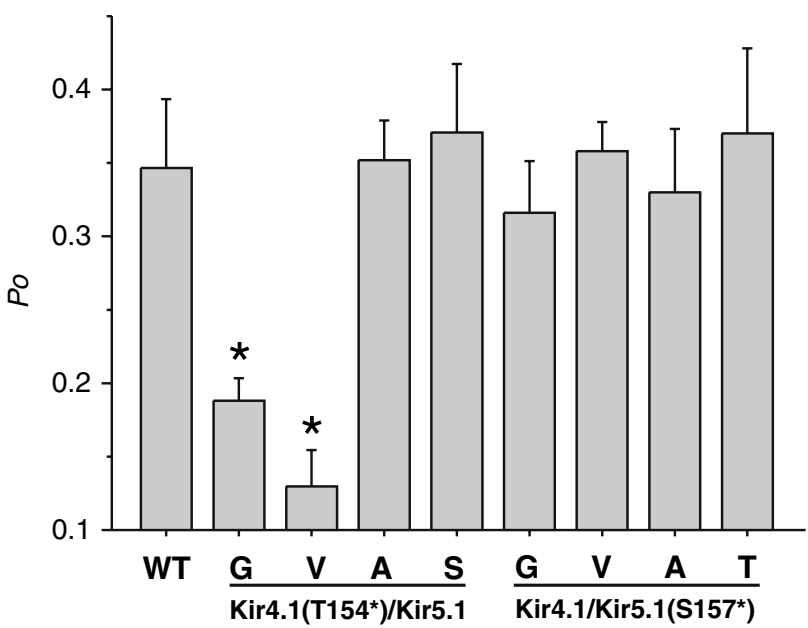

Fig. 4 The effect of different amino acid substitutions at the upper hinge residues (T154 in Kir4.1 subunit and S157 in Kir5.1 subunit) on single-channel open-probability $\left(P_{\mathrm{o}}\right)$ of heteromeric Kir4.1/Kir5.1 channels. $W T$ wild-type Kir4.1/Kir5.1. Currents were measured in the cell-attached mode at $-120 \mathrm{mV}, n=6 . * P<0.005$
These results indicate that contrary to the effect seen in KCNQ1 channels, mutation of the non-conserved "upper" gating hinge residues in Kir4.1 and Kir5.1 to a glycine residue does not increase the ability of the channel to open. Importantly, there is also a non-equivalent contribution of the two subunits to the gating process, with mutation of the Kir5.1 upper gating hinge residue having little or no effect, whilst similar mutations in the Kir4.1 subunit have a more significant and dominant effect.

Another unique characteristic of heteromeric Kir4.1/ Kir5.1 channels is their slow time-dependent activation at hyperpolarising potentials (Pessia et al. 1996). We therefore tested the effect of these mutations on this activation process. Table 2 shows that mutations at the upper hinge position in the Kir4.1 subunit showed significantly reduced $\tau$ values compared to wild-type Kir4.1/Kir5.1. By contrast, mutations at the upper hinge position in the Kir5.1 subunit showed no significant changes. However, despite this apparent correlation, there is evidence that this activation process reflects a slow "unblock" by intracellular polyamines (Lancaster et al. 2000). The dominant effect of mutations in Kir4.1 on this activation process may possibly be explained by the fact that only the Kir4.1 subunit contains a negatively charged residue at the TM2 rectification control site (E158), which binds polyamines deep within the pore (Fakler et al. 1994; Lu and MacKinnon 1994). According to our structural model (see below), this residue is placed one $\alpha$-helical turn below T154 in Kir4.1. Therefore, mutations at this position in Kir4.1 may have an indirect effect on channel activation that is unrelated to the relative flexibility of the helix at this position.

A recent study, which addressed the role of the upper TM2 gating hinge in Kir3.4, suggested that TM2 actually bends at the residue that precedes the upper glycine hinge, rather than at the glycine itself (Rosenhouse-Dantsker and Logothetis 2006). We therefore examined the effect of glycine substitutions adjacent to T154 in Kir4.1 and S157 in Kir5.1. We found that contrary to the effects observed in Kir3.4, Kir4.1(L153G)/Kir5.1 channels were similar to wild-type, whilst Kir4.1(T155G)/Kir5.1 channels showed a $63 \pm 11 \%(n=18)$ increase in whole-cell currents (Fig. 5). However, for Kir5.1, these positional effects were reversed; Kir4.1/Kir5.1(L156G)) showed a $44 \pm 9 \% \quad(n=18)$ increase in channel activity, whilst the Kir4.1/Kir5.1 (C158G) mutation had little effect (Fig. 5).

Because the effect of mutations in the Kir4.1 subunit were opposite to that observed in homomeric Kir3.4, we examined the effect of the activatory $\mathrm{T} 155 \mathrm{G}$ mutation in homomeric Kir4.1 channels and found that homomeric Kir4.1(T155G) still had an enhanced $P_{\mathrm{o}}(0.75 \pm 0.06$, $n=6)$ compared to wild-type Kir4.1 $(0.41 \pm 0.05, n=6)$. The effects of similar mutations in Kir5.1 were not exam- 
Table 2 Time constant values $(\tau)$ for time-dependent activation of different mutations at the upper Glycine hinge in heteromeric Kir4.1/Kir5.1 channels

\begin{tabular}{lllr}
\hline & $\tau(\mathrm{s})$ & & $\tau(\mathrm{s})$ \\
\hline Kir4.1/Kir5.1 & $1.63 \pm 0.16$ & & \\
Kir4.1(T154G)/Kir5.1 & $0.53 \pm 0.02^{* *}$ & Kir4.1/Kir5.1(S157G) & $1.59 \pm 0.15$ \\
Kir4.1(T154V)/Kir5.1 & $0.82 \pm 0.02^{* *}$ & Kir4.1/Kir5.1(S157V) & $1.27 \pm 0.05$ \\
Kir4.1(T154S)/Kir5.1 & $0.79 \pm 0.09^{* *}$ & Kir4.1/Kir5.1(S157T) & $1.25 \pm 0.10$ \\
Kir4.1(T154A)/Kir5.1 & $0.72 \pm 0.07^{* *}$ & Kir4.1/Kir5.1(S157A) & $1.31 \pm 0.04$ \\
\hline
\end{tabular}

The activation curves at $-120 \mathrm{mV}$ were fitted with a single exponential function

** $P$ value $<0.05$

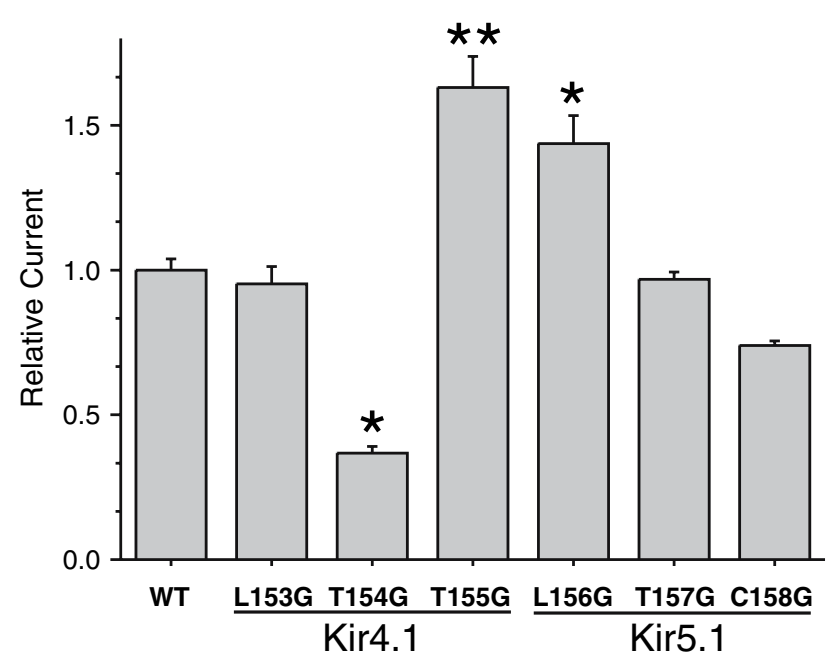

Fig. 5 Effect of glycine substitutions at positions adjacent to the upper hinge position on whole-cell currents of heteromeric Kir4.1/Kir5.1 channels. WT wild-type Kir4.1/Kir5.1. Maximal steady-state currents were recorded at $-120 \mathrm{mV}, n=12 . * P<0.005 . * P<0.05$

ined as homomeric Kir5.1 is non-functional. However, these results demonstrate that unlike Kir3.4, the gating hinge in Kir4.1 is unlikely to precede T154, and that TM2 flexibility may be more important at the subsequent amino acid (T155), which is below the proposed upper gating hinge.

The lower glycine hinge

The "lower" conserved glycine residue in TM2 at the helixbundle crossing is more highly conserved than the upper glycine and is invariant in all known mammalian Kir channel sequences. We therefore examined the functional effect of mutations at this "lower" putative glycine hinge position. Due to the location of this residue close to the intracellular gate of the channel, we made the conservative mutation of glycine to alanine on the basis that significantly larger or charged side chains may have multiple effects. Surprisingly, we found that even this subtle glycine to alanine mutation had a profound effect on channel activity; for
Kir4.1(G163A)/Kir5.1 channels, whole cell currents were reduced by $39 \pm 4 \%(n=18)$ compared to wild-type, and Kir4.1/Kir5.1(G166A) currents were reduced by $71 \pm 2 \%$ $(n=18)$. Such profound effects of a subtle mutation at this lower glycine position may explain why it is so highly conserved throughout the Kir channel superfamily and indicate a high degree of structural and/or functional importance for this residue.

\section{Structural model of heteromeric Kir4.1/Kir5.1}

To understand the structural basis of these results, we made use of a homology model of heteromeric Kir4.1/Kir5.1 (Shang et al. 2005). This model shows that instead of being isolated within TM2, both "upper hinge" residues (T154 in Kir4.1 and S157 in Kir5.1) are in direct contact with residues at the base of the pore $\alpha$-helix, which precedes the selectivity filter (Fig. 6).

In Kir3.4, it has been proposed that the small volume of the glycine side chain at this upper hinge position may be as important as its effect on TM2 flexibility in order to prevent possible "constraining interactions" with residues in the selectivity filter (Rosenhouse-Dantsker and Logothetis 2006). Our results with T154 in Kir4.1 show that side chains with only a slightly smaller volume to Thr (i.e. Ser, Ala) have little effect on $P_{\mathrm{o}}$, but that changes to substantially larger (Val) or substantially smaller (Gly) residues have a profound effect. This suggests that rather than avoiding any "constraining" interaction with the pore helix, this "hinge residue" directly contacts the pore-helix, and that altering the optimal nature of this interaction by introducing either larger, or smaller side chains impairs channel opening. It is particularly interesting that the effects of these mutations have a dominant effect in Kir4.1, whereas they have little effect in Kir5.1 (Fig. 5). This may provide some insight into the functional contribution of different subunits within novel heteromeric channels such as Kir4.1/Kir5.1 and demonstrate the non-equivalent role of these two subunits to the gating process. The important nature of the interaction between the residue at the upper hinge position 

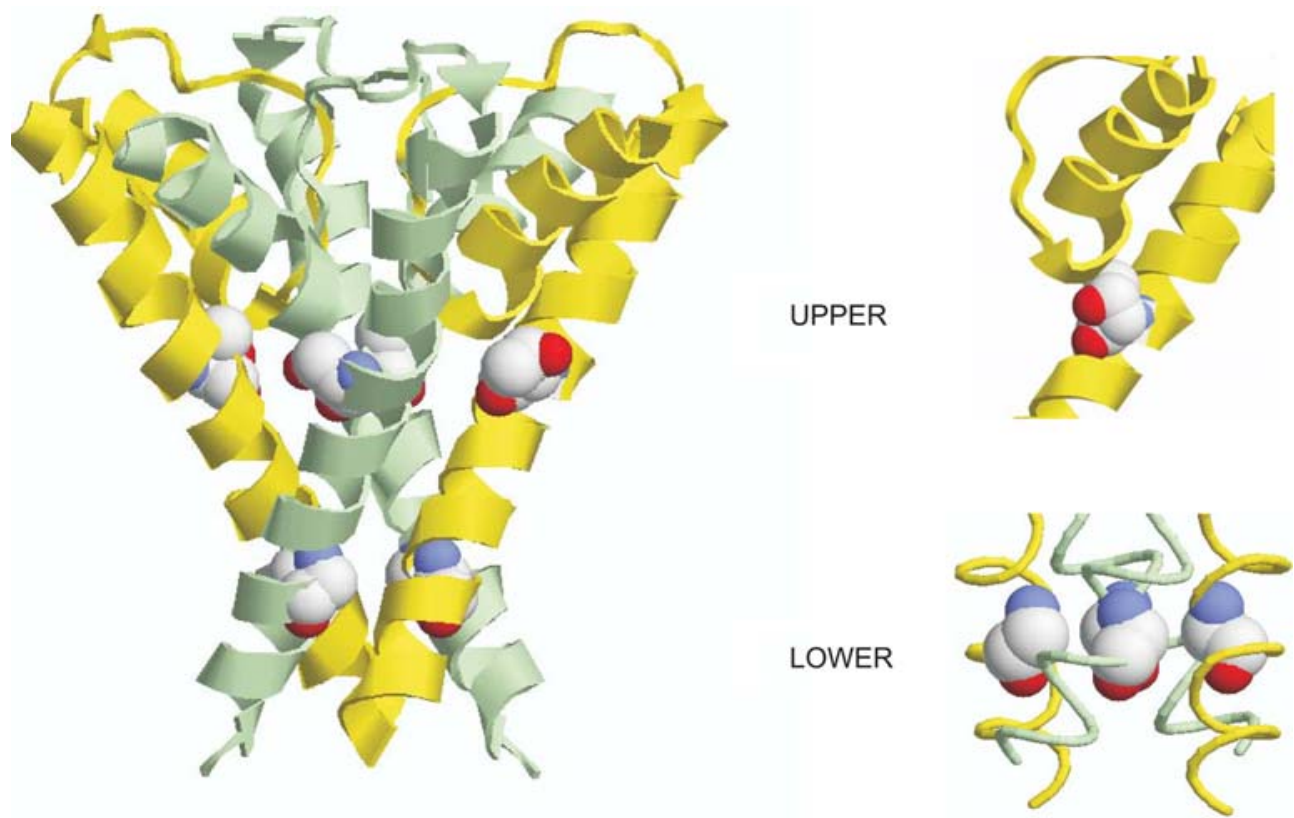

Fig. 6 Homology model of heteromeric Kir4.1/Kir5.1. For clarity, only TM2 and the pore $\alpha$-helices are shown. Kir4.1 TM2 is shown in yellow, Kir5.1 TM2 in light blue. The upper hinge residues (Kir4.1T154, Kir5.1-S157) and the putative lower hinge glycines (Kir4.1-

in Kir4.1 and the pore $\alpha$-helix may also be part of a structural mechanism by which movement in the TM domains communicates with the selectivity filter.

Although the primary gate in $\mathrm{K}^{+}$channels is thought to be at the helix-bundle crossing, several elegant studies have now demonstrated that an additional "gate" resides within the selectivity filter of the $\mathrm{K}^{+}$channel pore, and a "filter gate" has been proposed to exist in the related Kir6.2 channel (Berneche and Roux 2005; Cordero-Morales et al. 2006; Proks et al. 2003). It is also now becoming clear that mutations within the TM helices of several different Kir channels can influence the properties of the selectivity filter and that some degree of allosteric "communication" exists between them (Bichet et al. 2004; Rapedius et al. 2006). Instead of simply affecting TM2 flexibility, mutations at the upper hinge position may therefore disrupt the optimal nature of the interaction between this residue in Kir4.1 and the pore $\alpha$-helix, and thereby the communication between the TMs and any putative gate that resides within the filter of Kir4.1. More detailed studies to address the specific contribution of TM2 flexibility at this upper hinge position to Kir channel gating may require novel approaches, such as unnatural amino acid mutagenesis of the peptide backbone, in order to exclude the secondary effects of mutating TM2 side chains to other residues, such as prolines and glycines used in previous studies (Jin et al. 2002; RosenhouseDantsker and Logothetis 2006).

It may not be inconsequential that only Kir4.1, Kir4.2 and Kir5.1 have a non-conserved residue at the upper hinge
G163, Kir5.1-G166) are indicated as $v d w$ spheres in $c p k$. Enlarged views of the upper hinge area of Kir4.1 showing T154 and of the lower hinge areas are shown on the right hand side

position because these subunits exhibit highly selective heteromeric coassembly with each other. Kir5.1 only physically assembles with members of the Kir4.0 subfamily (Konstas et al. 2003; Pessia et al. 2001). It is possible that such differences in a highly conserved sequence motif contribute to the highly selective heteromultimerisation between these subunits, as well as to their functional properties.

Examination of the homology model also shows that the lower highly conserved glycine residue occurs within the helix-bundle crossing and that the small size of this side chain appears essential to allow tight packing of the helixbundle crossing. Even modest mutations at this position such as glycine to alanine are likely to disrupt packing of the TM2 helices at this point, and this could easily explain the effects on channel activity seen in these mutants. Furthermore, in the closed state crystal structure of KirBac1.1, and the partially open state crystal structure of KirBac3.1, the TM2 helices are not kinked at this position and our data support current models, which suggest that this part of TM2 moves as a rigid body during channel opening (Kuo et al. 2003). Therefore, the very highly conserved glycine found at this position in all Kir channels is probably to allow packing of the helices at this point, rather than to act as a lower "hinge" during channel gating.

In conclusion, we have taken advantage of the non-conserved nature of the upper TM2 glycine hinge residue in heteromeric Kir4.1/Kir5.1 channels to show that the relationship between the putative "flexibility" of this hinge and channel 
opening is more complex than originally thought, as this hinge residue makes direct contact with structures within the filter region. Therefore, the effect of mutations on TM2 flexibility are not easily separated from secondary effects they may have on gating elements contained within the selectivity filter. Furthermore, we found that individual subunits within this heteromeric Kir4.1/Kir5.1 channel contribute differently to this gating process. This asymmetric behaviour may have important consequences for understanding the functional contribution of individual subunits within other heteromeric Kir channels (e.g. Kir3/GIRK channels). Importantly, it may also reflect part of the structural mechanisms, which contribute to the highly selective assembly and heteromultimerisation that occurs between Kir5.1 and Kir4.1 subunits.

Acknowldegments This work was supported by grants from the Royal Society and The Wellcome Trust. SJT is a Royal Society University Research Fellow.

\section{References}

Berneche S, Roux B (2005) A gate in the selectivity filter of potassium channels. Structure 13:591-600

Bichet D, Lin YF, Ibarra CA, Huang CS, Yi BA, Jan YN, Jan LY (2004) Evolving potassium channels by means of yeast selection reveals structural elements important for selectivity. Proc Natl Acad Sci U S A 101:4441-4446

Bright JN, Shrivastava IH, Cordes FS, Sansom MS (2002) Conformational dynamics of helix S6 from Shaker potassium channel: simulation studies. Biopolymers 64:303-313

Casamassima M, D’Adamo MC, Pessia M, Tucker SJ (2003) Identification of a heteromeric interaction that influences the rectification, gating, and $\mathrm{pH}$ sensitivity of Kir4.1/Kir5.1 potassium channels. J Biol Chem 278:43533-43540

Cordero-Morales JF, Cuello LG, Zhao Y, Jogini V, Cortes DM, Roux B, Perozo E (2006) Molecular determinants of gating at the potassium-channel selectivity filter. Nat Struct Mol Biol 13:311-318

Cui N, Giwa LR, Xu H, Rojas A, Abdulkadir L, Jiang C (2001) Modulation of the heteromeric Kir4.1-Kir5.1 channels by $\mathrm{PCO}_{2}$ at physiological levels. J Cell Physiol 189:229-236

Derst C, Karschin C, Wischmeyer E, Hirsch JR, Preisig-Muller R, Rajan S, Engel H, Grzeschik K, Daut J, Karschin A (2001) Genetic and functional linkage of Kir5.1 and Kir2.1 channel subunits. FEBS Lett 491:305-311

Ding S, Ingleby L, Ahern CA, Horn R (2005) Investigating the putative glycine hinge in Shaker potassium channel. J Gen Physiol 126:213-226

Doyle DA (2004) Structural changes during ion channel gating. Trends Neurosci 27:298-302

Doyle DA, Morais Cabral J, Pfuetzner RA, Kuo A, Gulbis JM, Cohen SL, Chait BT, MacKinnon R (1998) The structure of the potassium channel: molecular basis of $\mathrm{K}^{+}$conduction and selectivity. Science 280:69-77

Fakler B, Brandle U, Bond C, Glowatzki E, Konig C, Adelman JP, Zenner HP, Ruppersberg JP (1994) A structural determinant of differential sensitivity of cloned inward rectifier $\mathrm{K}^{+}$channels to intracellular spermine. FEBS Lett 356:199-203

Jiang Y, Lee A, Chen J, Cadene M, Chait BT, MacKinnon R (2002) The open pore conformation of potassium channels. Nature 417:523-526
Jin T, Peng L, Mirshahi T, Rohacs T, Chan KW, Sanchez R, Logothetis DE (2002) The ( $\beta \gamma$ subunits of $\mathrm{G}$ proteins gate a $\mathrm{K}^{+}$channel by pivoted bending of a transmembrane segment. Mol Cell 10:469481

Konstas AA, Korbmacher C, Tucker SJ (2003) Identification of domains that control the heteromeric assembly of Kir5.1/Kir4.0 potassium channels. Am J Physiol Cell Physiol 284:C910C917

Kuo A, Gulbis JM, Antcliff JF, Rahman T, Lowe ED, Zimmer J, Cuthbertson J, Ashcroft FM, Ezaki T, Doyle DA (2003) Crystal structure of the potassium channel KirBac1.1 in the closed state. Science 300:1922-1926

Lancaster MK, Dibb KM, Quinn CC, Leach R, Lee JK, Findlay JB, Boyett MR (2000) Residues and mechanisms for slow activation and $\mathrm{Ba}^{2+}$ block of the cardiac muscarinic $\mathrm{K}^{+}$channel, Kir3.1/ Kir3.4. J Biol Chem 275:35831-35839

Long SB, Campbell EB, Mackinnon R (2005) Crystal structure of a mammalian voltage-dependent Shaker family $\mathrm{K}^{+}$channel. Science 309:897-903

Lu Z, MacKinnon R (1994) Electrostatic tuning of $\mathrm{Mg}^{2+}$ affinity in an inward-rectifier $\mathrm{K}^{+}$channel. Nature 371:243-246

Magidovich E, Yifrach O (2004) Conserved gating hinge in ligandand voltage-dependent $\mathrm{K}^{+}$channels. Biochemistry 43:1324213247

Perozo E, Cortes DM, Cuello LG (1999) Structural rearrangements underlying $\mathrm{K}^{+}$-channel activation gating. Science 285:73-78

Pessia M, Imbrici P, D’Adamo MC, Salvatore L, Tucker SJ (2001) Differential pH sensitivity of Kir4.1 and Kir4.2 potassium channels and their modulation by heteropolymerisation with Kir5.1. J Physiol 532:359-367

Pessia M, Tucker SJ, Lee K, Bond CT, Adelman JP (1996) Subunit positional effects revealed by novel heteromeric inwardly rectifying $\mathrm{K}^{+}$channels. EMBO J 15:2980-2987

Proks P, Antcliff JF, Ashcroft FM (2003) The ligand-sensitive gate of a potassium channel lies close to the selectivity filter. EMBO Rep 4:70-75

Rapedius M, Haider S, Browne KF, Shang L, Sansom MS, Baukrowitz T, Tucker SJ (2006) Structural and functional analysis of the putative $\mathrm{pH}$ sensor in the Kir1.1 (ROMK) potassium channel. EMBO Rep 7:611-616

Rosenhouse-Dantsker A, Logothetis DE (2006) New roles for a key glycine and its neighboring residue in potassium channel gating. Biophys J 91:2860-2873

Seebohm G, Strutz-Seebohm N, Ureche ON, Baltaev R, Lampert A, Kornichuk G, Kamiya K, Wuttke TV, Lerche H, Sanguinetti MC, Lang F (2006) Differential roles of S6 domain hinges in the gating of KCNQ potassium channels. Biophys J 90:2235-2244

Shang L, Lucchese CJ, Haider S, Tucker SJ (2005) Functional characterisation of missense variations in the Kir4.1 potassium channel (KCNJ10) associated with seizure susceptibility. Brain Res Mol Brain Res 139:178-183

Tucker SJ, Imbrici P, Salvatore L, D'Adamo MC, Pessia M (2000) pH dependence of the inwardly rectifying potassium channel, Kir5.1, and localization in renal tubular epithelia. J Biol Chem 275:16404-16407

Wu J, Xu H, Shen W, Jiang C (2004) Expression and coexpression of $\mathrm{CO}_{2}$-sensitive Kir channels in brainstem neurons of rats. J Membr Biol 197:179-191

Yang Z, Xu H, Cui N, Qu Z, Chanchevalap S, Shen W, Jiang C (2000) Biophysical and molecular mechanisms underlying the modulation of heteromeric Kir4.1-Kir5.1 channels by $\mathrm{CO}_{2}$ and $\mathrm{pH}$. J Gen Physiol 116:33-45

Zhao Y, Yarov-Yarovoy V, Scheuer T, Catterall WA (2004) A gating hinge in $\mathrm{Na}^{+}$channels; a molecular switch for electrical signaling. Neuron 41:859-865 